\title{
Predictive Control of a Closed Grinding Circuit System in Cement Industry
}

\author{
Luis I. Minchala ${ }^{1,3}$, Member, IEEE, Youmin Zhang ${ }^{2}$, Senior Member, IEEE, Luis E. Garza-Castañón ${ }^{3}$, Member, \\ IEEE
}

\begin{abstract}
This paper presents the development of a non-linear model predictive controller (NMPC) applied to a closed grinding circuit system in the cement industry. A Markov chain model is used to characterize the cement grinding circuit by modeling the ball mill and the centrifugal dust separator. The probability matrices of the Markovian model are obtained through a combination of comminution principles and experimental data obtained from the particle size distribution (PSD) of cement samples at specific stages of the system. The NMPC is designed as a supervisory controller in order to manage distributed controllers (DCs) installed in the process. Both the model and the controller are validated online through the implementation of the proposed approach in the supervisory control and data acquisition (SCADA) system of an industrial plant. The results show a significant improvement in the performance of the grinding circuit in comparison to the operation of the system without the proposed controller.
\end{abstract}

Index Terms-Grinding circuit, Markov chain, NMPC

\section{INTRODUCTION}

The cement manufacturing process is composed by three main stages: i) raw materials extraction, ii) blending and clinkerization, and iii) grinding and distribution. Cement manufacturing is highly energy demanding, and is dependent on the availability of natural resources. Typically, the consumption in a modern cement plant is between 110 and $120 \mathrm{kWh}$ per ton of produced cement [1]. The grinding stage represents about $40 \%$ of the total electrical energy consumption of the cement manufacturing. Therefore, the industry is continuously focusing on reducing the consumption of natural resources and energy at the manufacturing and operational stages [2].

Cement grinding is responsible for the fineness characteristics of the final product, typically measured by two quality variables: specific surface or Blaine $\left(\mathrm{cm}^{2} / \mathrm{g}\right)$, and the percentage of material passing the sieve No. 325 (PPS325). It is necessary to develop innovative tools for an enhanced operation of the cement grinding circuit, which allow the minimization of the final product variability, while reducing the energy consumption at this stage.

Many contributions have been made in the control of grinding circuits. For instance, reference [3] proposes a dynamical model of the grinding process to tune the PID controllers involved in the cement milling by the loop shaping method.

\footnotetext{
${ }^{1}$ Universidad de Cuenca, Av. 12 de Abril, Cuenca, Ecuador

2 Concordia University, 1455 de Maisonneuve W., Montreal QC, Canada (Corresponding author; Email: youmin.zhang@concordia.ca)

3 Tecnológico de Monterrey, Campus Monterrey, Eugenio Garza Sada 2501, Monterrey NL, México
}

The authors of [4] present a modeling procedure of process variables (PV) to design a linear model predictive controller (MPC) for the operation of a grinding circuit of a cement plant. The modeling procedure is based on the step-response analysis of certain operation variables of the process. The proposed approach is compared with a knowledge-based fuzzy control system, offering better results in the handling of uncertainties, and also presenting less variation in the manipulated variables (MVs). Reference [5] proposes a fuzzy approach to control the process, based on operation rules of the grinding circuit. Reference [6] proposes an intelligence-based supervisory control for a grinding circuit, which combines an artificial neural networkbased soft-sensor, a fuzzy logic-based dynamic adjustor, and an expert-based overload diagnosis and adjustment module. This automatic system adjusts the set-points of DCs in the grinding circuit eliminating the need of operators.

In [7] a non-linear model of the grinding circuit for predicting the flow rates of the materials is proposed in order to design an NMPC. Simulation results validate the performance of the controller. Reference [8] presents a framework for modeling hybrid systems and for developing hybrid predictive controllers. This approach is tested in two simulated applications, crushing circuits and flotation. The results are compared with conventional MPC controllers, which show better performance of the hybrid controller. Reference [9] proposes a grinding particle size prediction algorithm using neural networks, and a data-driven optimization control approach to guarantee safe operation and overload fault prevention of a hematite grinding process. The proposed control system is tested in a hardwarein-the-loop experimental system used to demonstrate optimal operation of the grinding process.

The majority of papers in this research field present simulation-based results. Few authors provide experimental results obtained with advanced controllers applied to actual industrial grinding circuits. Lepore et al. [10], [11] used a distributed reduced-order model for the design of an NMPC applied to a cement mill circuit. The control variable is the PSD of the cement product resulting from the manipulation of the fresh-feed flow rate and the separator speed. The objective function is selected to perform a classic DMC control strategy, essentially adjusting the bias between measured and predicted outputs assumed to be caused by step disturbances, which is not necessarily true in industrial grinding circuits. The present research proposes a Markovian model to deal with this issue.

In the present work an implementation of an NMPC system of a cement grinding circuit in an operating industrial plant, based on a stochastic model of the process, is demonstrated. 
This paper presents the modeling of a closed grinding circuit system and the design of a predictive supervisory control strategy installed in the SCADA system of the grinding circuit of the cement plant. A hidden Markov chain model mimics the dynamics of the cement mill and the dust separator. With the help of this model, an NMPC control system was designed and applied to the closed-loop grinding circuit. The probability matrices of the Markovian model are obtained from a combination of comminution principles and experimental data obtained from the PSD of the product streams at specific stages of the system. The NMPC manipulates the set points of DCs installed in the process. The model and the controller are validated online through the implementation of the proposed approach in the SCADA system. The results show significant improvements in the grinding circuit stability and productivity. Prior to the implementation of the proposed controller, the set points of the DCs of the grinding circuit system were operated manually.

\section{GRINDING CIRCUIT OPERATION}

The grinding is mostly performed in closed circuits where a horizontal ball cement mill is fed with raw materials. The fresh flow feed is composed of clinker, gypsum and other components like limestone, pozzolans, and fly ash. The milled product is fed via a recycle elevator to a dynamic separator. The high fineness stream of the separator constitutes the final circuit product and is sent to the storage and dispatch silos, while the coarse material returns back to the cement mill to be ground again. Fig. 1 shows the grinding circuit layout of the industrial cement plant under study. The elements identified in the cement grinding circuit are:

- G20 is the ball mill;

- G24 is the dust collector of the ball mill;

- G31 is the bucket elevator;

- G35 is the centrifugal separator; and

- G39 is the dust collector of the separator.

The grinding circuit is operated from a control panel through a SCADA system. The operators manipulate four variables:

- Fresh feed flow $(t p h)$. This set point changes the speed of the conveyors and the dosage of the storage silos containing the raw material, in a coordinated way.

- G24 speed (rpm). This control signal has a direct impact on the residence time of the mill. The speed of this dust collector is kept constant most of the times.

- G35 speed (rpm). The rotor speed of the separator is responsible for producing centrifugal forces in the cement particles, which in combination with the particles weight and the drag forces produced by the dust collector G39, completes the classification process.

- G39 speed (rpm). The cement fineness depends on the speed of this dust collector. Therefore, G35 and G39 speeds are the variables that are to be manipulated in order to stabilize the material flows in the grinding circuit to obtain desired cement fineness.

Fig. 2 shows the open-loop operation of the grinding circuit during two days of January-2015. The MVs managed from the SCADA system can be seen to have a direct influence on the

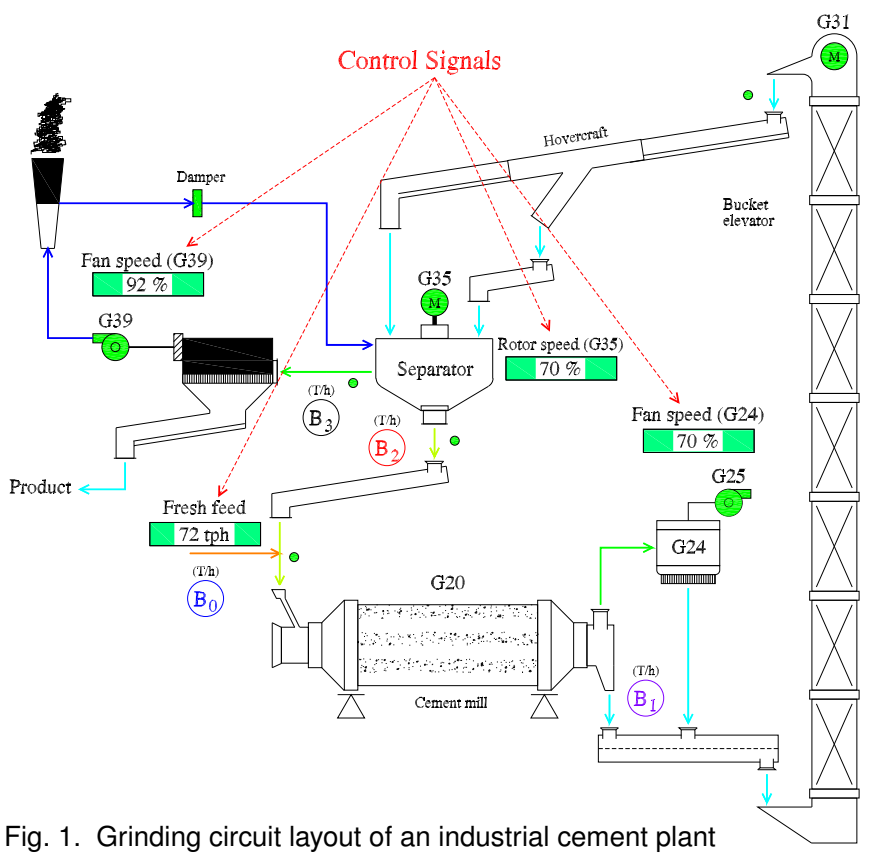

dynamics of the system. Speed changes in the G35 and G39 units produce instantaneous changes in the reject flow, while a step change in the fresh feed flow produces a response in the reject flow with a characteristic time constant of eight minutes, which is the mill residence time. The reject flow presents a high variation range, which is responsible also for high fineness variations of the final product. The objective of the present work was to develop a control strategy to stabilize the reject flow signal and to maximize the productivity, while keeping the cement fineness variability within the limits set by the product specifications.

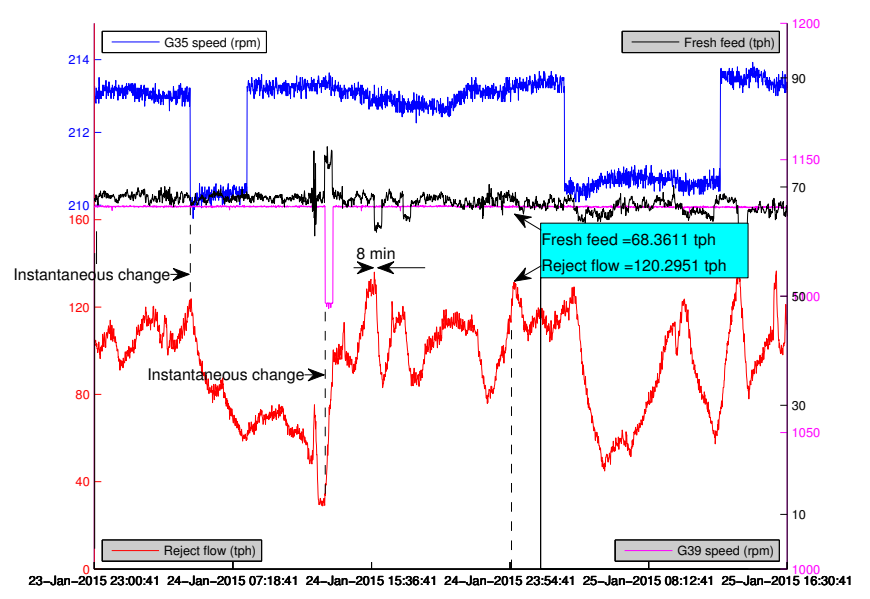

Fig. 2. Grinding circuit operation during forty eight hours

\section{MOdELING OF THE GRINDING CIRCUIT}

Modeling of an industrial milling system is a complicated task due to the multivariable character of the process, the high degree of load disturbances, the incomplete or missing 
information about some key process characteristics such as clinker hardness, the moisture of the raw material, grinding media condition, separator efficiency and the PSD of different streams. Therefore, for designing a model-based control of a cement mill, a modeling procedure with a stochastic behavior is needed. The Markov chain approach is ideally suited for this purpose, where the probability of a data set within a defined state space is easily obtained from experimental data.

A Markov chain of a random process with finite state space $\left(S=\left\{s_{1}, \ldots, s_{k}\right\}\right)$ is defined as [12]:

$$
\begin{gathered}
\mathbf{P}\left(\mathbf{X}_{k+1}=s_{j} \mid \mathbf{X}_{0}=s_{1_{0}}, \mathbf{X}_{1}=s_{i_{1}}, \ldots, \mathbf{X}_{k}=s_{i}\right) \\
=\mathbf{P}\left(\mathbf{X}_{k+1}=s_{j} \mid \mathbf{X}_{k}=s_{i}\right)=P_{i j}
\end{gathered}
$$

where $\mathbf{P} \in \mathbb{R}^{k \times k}$ is the transition matrix. The transition probability $P_{i j}$ is the conditional probability of being in state $s_{j}$ given that the previous state is $s_{i}$.

For a Markov chain with state space $\left\{s_{1}, \ldots, s_{k}\right\}$, an initial state vector $x(0)$, and transition matrix $\mathbf{P}$, the evolution is:

$$
x(k)=x(0) \mathbf{P}^{k}
$$

The grinding circuit is modeled with the use of Markov chains, considering the methodology presented in [13]. Fig. 3 shows a block diagram of the model to be developed. The grinding circuit model describes a finite state space, $S=\left\{s_{1}, \ldots, s_{42}\right\}$, where $s_{i}$ represents a computational sieve with a defined cement fineness. The PSD of the fresh feed material is coarser than other flows in the grinding circuit. The differences in the sieve sizes used in the PSD streams of the grinding circuit, e.g. the fines stream is finer than other streams in the grinding circuit, are solved by defining the PSD data over the same set of sieves. The set of computational sieves cover the entire range of particle sizes in the PSD, from the smallest in the final product to the largest in the fresh feed. The following definition of computational sieves was used for all particle streams:

$$
x m_{i}=x m_{\min }+\gamma 2^{\frac{i-2}{2}}
$$

where,

$$
\begin{aligned}
& x m_{i} \quad-\text { Computational sieves set }(\mu m) \\
& x m_{\text {min }}-\text { Smallest computational sieve }(i=1) \\
& i \quad-\quad \text { Number of the sieve, } i=1,2, \ldots, 42 \\
& \gamma \quad-\text { Free parameter, }
\end{aligned}
$$

The state vector of the model corresponds to the PSD of the material flow in three different stages of the grinding circuit: the mill output flow $\left(\mathbf{f}_{1} \in \mathbb{R}^{42}\right)$, reject flow $\left(\mathbf{f}_{2} \in \mathbb{R}^{42}\right)$, and fines flow $\left(\mathbf{f}_{3} \in \mathbb{R}^{42}\right)$; the PSD of the material streams are calculated through two probability matrices, the grinding matrix $\left(\mathbf{G} \in \mathbb{R}^{42 \times 42}\right)$, and the classification matrix $\left(\mathbf{C} \in \mathbb{R}^{42 \times 42}\right)$. The initial state vector $\left(\mathbf{f}_{0} \in \mathbb{R}^{42}\right)$ represents the PSD of the fresh feed.

The conditional probabilities of the matrices $\mathbf{G}$ and $\mathbf{C}$ are obtained through a combination of data analysis of measured PSDs of the streams in the grinding circuit and comminution principles. The green dots in Figs. 1 and 3 represent sampling points, where samples of the material flowing through

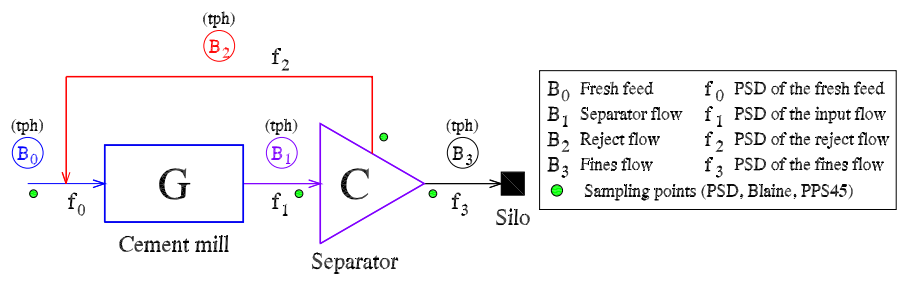

Fig. 3. Block diagram of the Markovian model of the grinding circuit

the grinding circuit are taken to be analyzed by a particle sizing instrument, model MASTERSIZER 3000. Fig. 4 shows representative PSD of the streams of the grinding circuit at the sampling points. The difference in particle size between the different streams can be clearly appreciated.

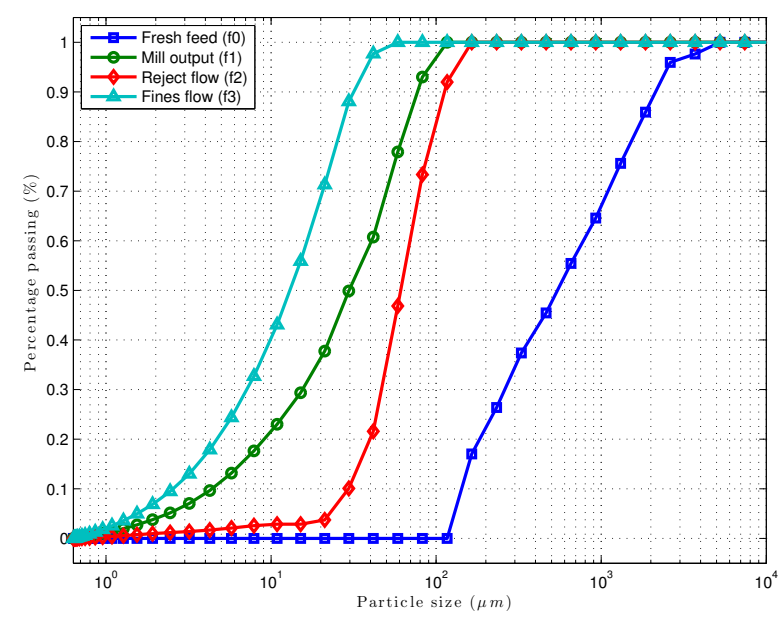

Fig. 4. PSD of the grinding circuit streams

\section{A. Estimation of the grinding matrix}

The grinding forces produce clinker particles to fracture with some probability. Once the particle is broken, it does not belong to the original fraction (source sieve $_{j}$ ) and their pieces are distributed among fractions of smaller size (receptor sieve $\left._{i}\right)$. The amount of ground material and the way the particles are propagated between smaller size fractions are determined through the selection and distribution functions [14]. The selection function describes the breakage rate of a particle; its units are $1 /$ second. This function is a representation of the grinding principles and relates the size of a particle subject to a given grinding force with the specific energy required to form a new particle surface. The selection function can be written as follows:

$$
s(x)=\alpha\left(\frac{x}{x_{\max }}\right)^{\beta}
$$

where,

- Original particle size before fracture

$x_{\max }$ - Largest computational sieve of the PSD $(\mu m)$

$\alpha \quad$ - Grinding constant

$\beta \quad-$ Grinding constant 
The result of multiplying $s(x)$ with an observation period $\Delta t$ in seconds defines the probability of a clinker particle to fracture. Therefore,

$$
P(x)=\Delta t s(x)
$$

Ground particles propagate among smaller size fractions according to the distribution law, represented by the parameter $b_{i j}$. The subindex $i$ represents the recepting fraction, while the subindex $j$ denotes the source fraction. The proportion of material that is transferred to the $i$-fraction is $\left(b_{i j} s_{j} \Delta t\right)$. The sum of the parameters $b_{i j}$ has to be 1 , which means that the amount of ground material from fraction $x m_{j}$ is exactly equal to the amount of material distributed among the smaller fractions $\left(\sum_{i=1}^{j-1} b_{i j}=1\right)$. The values of $b_{i j}$ are assumed to have the same probability for $j=1,2, \ldots, 42$, i.e. equal probabibilties for the original particle of size $x$ to break into particles of any of the smaller computational sieve classes.

The grinding matrix is a special case of transition matrix. It is derived by considering that the grinding process occurs in fundamental steps that represent breakage events [15]. One of those events corresponds to a unique transition of a Markov chain, and a repetitive process of this chain represents the transitions of state vector $\left(\mathbf{f}_{0} \rightarrow \mathbf{f}_{1}\right)$ in a period of time. The grinding matrix is defined as follows [16]:

$$
\mathbf{G}=\left[\begin{array}{ccccc}
1 & b_{12} s_{2} \Delta t & b_{13} s_{3} \Delta t & \ldots & b_{1 n} s_{n} \Delta t \\
0 & 1-s_{2} \Delta t & b_{23} s_{3} \Delta t & \ldots & b_{2 n} s_{n} \Delta t \\
0 & 0 & 1-s_{3} \Delta t & \ldots & b_{3 n} s_{n} \Delta t \\
\ldots & \ldots & \ldots & \ddots & \ldots \\
0 & 0 & 0 & \ldots & 1-s_{n} \Delta t
\end{array}\right]
$$

The grinding matrix is an upper triangular matrix. The main diagonal elements define the probabilities of the particles of remaining in their original fractions. The elements of the upper right of the matrix represent the distribution function and define the probability of transition of a particle from the source (column) to the recepting fraction (row). The elements of the lower left of the matrix are zeros, which denotes that this model does not allow agglomeration of the particles, i.e. source fractions can not become larger.

A grinding event is represented by:

$$
\begin{aligned}
{\left[\mathbf{f}_{0}\right]_{k+1} } & =[\mathbf{G}]\left[\mathbf{f}_{0}\right]_{k} \\
{\left[\mathbf{f}_{1}\right] } & =[\mathbf{G}]^{k}\left[\mathbf{f}_{0}\right]_{0}
\end{aligned}
$$

where,

$\left[\mathbf{f}_{1}\right] \quad$ - State vector of the separator feed flow

$\left[\mathbf{f}_{0}\right]_{k} \quad$ - Fresh feed vector after the $k$-th transtition

$[\mathbf{G}]^{k} \quad$ - Result of $k$ transitions of the grinding matrix

$\left[\mathbf{f}_{0}\right]_{0} \quad$ - Initial state vector of the fresh feed.

The number of transitions of the Markov chain, $k$, is directly related with the residence time in the ball mill, since every transition of the Markov chain was determined experimentally to last one second. The higher the residence time, the higher the number of transitions will be, and in consequence the stream leaving the mill will be finer.
The set of unknown parameters of the grinding matrix $(\alpha, \beta, k)$ are found by using parameter estimation and data adjustment. Eq. (8) is used to optimize the error between the estimation given by the Markovian model and a data set of experimental PSD of the stream $\mathbf{f}_{1}$.

The experimental data used for the parameters estimation of the model consists of 21 samples of the four material streams of the grinding circuit: fresh feed, mill output, reject flow, and fines flow. For every sample it was determined experimentally the following information:

- PSD

- Percentage of material passing the sieve No. 325

- Specific surface (Blaine) $\left[\mathrm{cm}^{2} / \mathrm{g}\right]$

Every sample was taken directly from the process and analyzed in the lab. Before taking any sample, a specific operating condition was set. For instance, the operating conditions as well as the lab results of five different samples are shown in Table I.

TABLE I

OPERATING CONDITIONS OF THE GRINDING CIRCUIT FOR THE SAMPLES USED FOR PARAMETER ESTIMATION

\begin{tabular}{cccccc}
\hline \multirow{2}{*}{ Test } & \multicolumn{2}{c}{ Manipulated variables } & \multicolumn{2}{c}{ Lab results } \\
& Feeding & G35 (rpm) & G39 (rpm) & PPS45 & Blaine \\
\hline 1 & 70.2 & 176 & 1113 & 5.4 & 4305 \\
5 & 73.0 & 182 & 1138 & 4.1 & 4147 \\
9 & 69.3 & 187 & 1113 & 2.7 & 4772 \\
17 & 80.0 & 194 & 1164 & 6.6 & 3574 \\
\hline
\end{tabular}

\section{B. Estimation of the classification matrix}

The classification matrix calculates from $\mathbf{f}_{1}$ the amount of material that returns to the grinding circuit, $\mathbf{f}_{2}$ (reject flow) and the amount of material that goes to the silos, $\mathbf{f}_{3}$ (fines flow). The classification matrix is represented through a diagonal matrix, C. Every element of the main diagonal $c_{i}$ represents the probability of the fraction $i$ to become the final product. The classification matrix is the result of discretizing the grade efficiency curve (GEC) of the separator [13]. The size of $\mathbf{C}$ is determined by the number of elements of the state vector $f_{1}$.

$$
\mathbf{C}=\left[\begin{array}{cccc}
c_{1} & 0 & \ldots & 0 \\
0 & c_{2} & \ldots & 0 \\
\ldots & \ldots & \ddots & \ldots \\
0 & 0 & \ldots & c_{42}
\end{array}\right]
$$

The grade efficiency curve can be calculated from the PSD data from the streams of the grinding circuit by using the following expression:

$$
G E C=\frac{f_{3 i}}{f_{1 i}} \frac{f_{1 i}-f_{2 i}}{f_{3 i}-f_{2 i}}
$$

where $f_{1 i}, f_{2 i}, f_{3 i}$ represent the probability of a particle having the size $x_{i}$ in the fresh feed, reject flow, and fines flow, respectively.

On the other hand, the Tromp curve, also called separation curve or selectivity curve, is typically used as a measure of the separator efficiency. The Tromp curve is defined as the inverse 


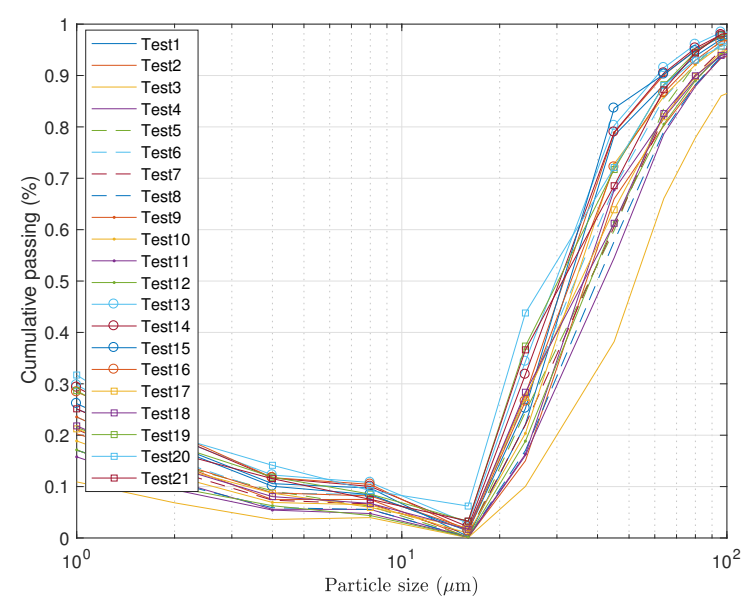

Fig. 5. Experimental Tromp curves for 21 different operating conditions

of the GEC; it models the probability of a particle entering the separator of becoming the final product. Since the separation process allows the modification of the separator rotor speed (G35), and the dust collector (G39) speed, the GEC will not be a static curve, and the $\mathbf{C}$ matrix depends on these two MVs. To handle the non-static behavior of $\mathbf{C}$, a neural network is designed to fit the data set from the experiments. Fig. 5 shows the experimental Tromp Curves of the 21 experimental data set used for the parameters estimation of the $\mathbf{G}$ and $\mathbf{C}$ matrices.

The PSD of $\mathbf{f}_{2}$ and $\mathbf{f}_{3}$ are calculated as follows:

$$
\begin{aligned}
{\left[\mathbf{f}_{2}\right] } & =([\mathbf{I}]-[\mathbf{C}])\left[\mathbf{f}_{1}\right] \\
{\left[\mathbf{f}_{3}\right] } & =[\mathbf{C}]\left[\mathbf{f}_{1}\right]
\end{aligned}
$$

where,

$\left[\mathbf{f}_{2}\right] \quad-\quad$ State vector of the reject flow

$\left[\mathbf{f}_{3}\right]_{k}-$ State vector of the fines flow

\section{Model validation}

Combining the submodels developed in the preceding sections the equation modeling the complete grinding circuit can now be written as follows:

$$
\left[\begin{array}{l}
\mathbf{f}_{1}(k+1) \\
\mathbf{f}_{2}(k+1) \\
\mathbf{f}_{3}(k+1)
\end{array}\right]=\left[\begin{array}{ccc}
\mathbf{0} & \mathbf{I}-\mathbf{C} & \mathbf{0} \\
\mathbf{G} & \mathbf{0} & \mathbf{0} \\
\mathbf{G} & \mathbf{C} & \mathbf{0}
\end{array}\right]\left[\begin{array}{c}
\mathbf{f}_{1}(k)+u_{3}(k) \\
\mathbf{f}_{2}(k) \\
\mathbf{f}_{3}(k)
\end{array}\right]
$$

It should be noticed that the $\mathbf{C}$ matrix is not constant and it rather depends on the MVs.

Fig. 6 shows the PSDs estimation of the grinding circuit streams by using Eq. (13). The mean squared error of the estimation of the mill output flow, $f_{1}$, is 0.0113 . The remaining two streams are calculated with the classification matrix $\mathbf{C}$. The estimation errors of the reject flow and fines flow are: error $_{f_{2}}=0.042$, and error $_{f_{2}}=0.142$.

\section{CONTROLleR DESIGN}

The grinding circuit at the cement plant has DCs, which are manipulated from the SCADA system. Therefore, the control

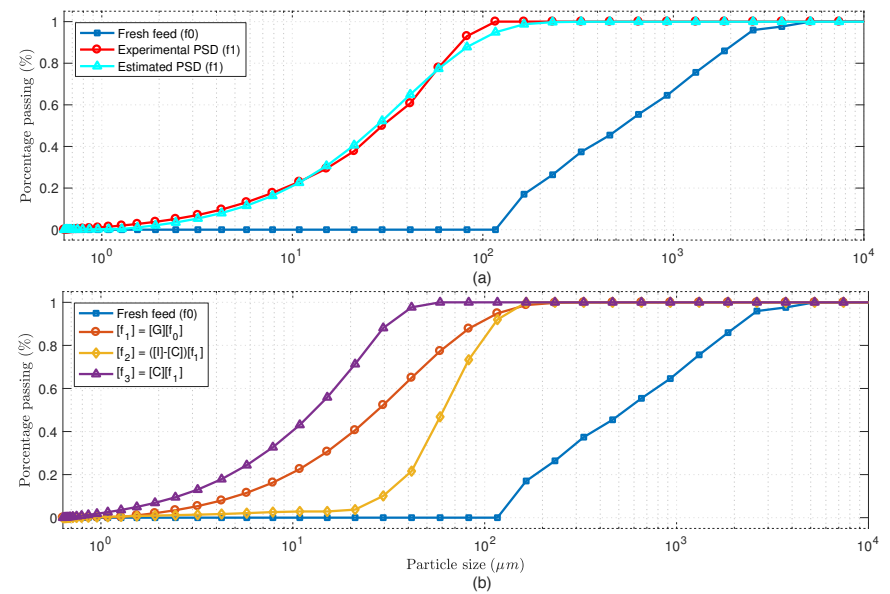

Fig. 6. (a) Mill output flow $\left(\mathbf{f}_{\mathbf{1}}\right)$ estimation by using the grinding matrix (b) Grinding circuit streams estimation by the Markovian model

strategy to be developed needs to manage the DCs in order to operate the grinding circuit. In this research work, the NMPC algorithm is selected due to its ability to focus on process variables (reject flow, Blaine, etc.) and its inherent possibility of sending set-points to process control loops.

\section{A. Nonlinear model predictive control}

NMPC is characterized by the use of nonlinear system models to predict and optimize the future system behavior [17]. Additionally, the method allows incorporating nonlinear constraints on the MVs, process variables (PVs), and states of the system. It is also possible to use cost functions different from the classic quadratic, since the optimization is performed over a finite-length horizon $(N)$. A generalized nonlinear discrete-time model of a system is defined as:

$$
\mathbf{x}_{k+1}=f\left(\mathbf{x}_{k}, \mathbf{u}_{k}\right)
$$

where $f: \mathbb{R}^{n} \times \mathbb{U}^{m} \rightarrow \mathbb{R}^{n}$ assigns $\mathbf{x}_{k+1} \in \mathbb{R}^{n}$ at the next time instant to each pair of state vector $\mathbf{x}_{k} \in \mathbb{R}^{n}$ and control signal vector $\mathbf{u}_{k} \in \mathbb{R}^{m}$.

The main objective is to control the state $\mathbf{x}_{k}$ of the system towards a reference trajectory $\mathbf{r}_{k}$ and to keep the system state close to this reference [18]. An equilibrium point of the closedloop system is defined by:

$$
\mathbf{x}_{k+1}^{*}=f\left(\mathbf{x}_{k}, \mu\left(\mathbf{x}_{k}\right)\right)
$$

where $\mu(\cdot)$ is the control law obtained with the NMPC algorithm.

A set of finite control sequences is obtained after the optimization process: $\mathbf{u}_{0}, \mathbf{u}_{1}, \ldots, \mathbf{u}_{N-1}$ for $N \in \mathbb{N}$ being the optimization horizon. Therefore, given an initial value $\mathbf{x}_{0} \in \mathbb{R}^{n}$ and a control sequence $\mathbf{u}(\cdot) \in \mathbb{U}^{K}$ a trajectory of Eq. (14) is obtained iteratively via:

$$
{ }^{u} \mathbf{x}_{k+1}=f\left({ }^{u} \mathbf{x}_{k}, \mathbf{u}_{k}\right)
$$

where ${ }^{u} \mathbf{x}_{k}$ is the predicted state of the system when the optimized control sequence $\mathbf{u}(\cdot)$ is applied. 
One of the strengths of MPC is its ability to manage constraints in its structure. It is necessary to define valid numerical sets for the states of the system, $\mathbf{x} \in \mathbb{X}_{x_{\min }}^{x_{\max }} \in \mathbb{R}^{n}$ and the control signals, $\mathbf{u} \in \mathbb{U}_{u_{\min }}^{u_{\max }} \in \mathbb{R}^{m}$.

\section{B. NMPC design}

The NMPC processes a data set of measurements from the grinding circuit in order to predict the system behavior through the Markovian model represented by Eq (13). Once the NMPC algorithm finds the optimal control vector, $\mathbf{u}^{*}=$ $\left[\begin{array}{lll}u_{1}^{*} & u_{2}^{*} & u_{3}^{*}\end{array}\right]^{T}=\left[\begin{array}{lll}G 35_{\text {speed }}^{*} & G 39_{\text {speed }}^{*} & B 0_{t p h}^{*}\end{array}\right]^{T}$, this information is sent to the DCs.

The NMPC algorithm runs in an OPC (formally known as Object Linking and Embedding for Process Control) client software, for this particular case we have selected MATLAB, which interchanges information with the SCADA system to write the control vector as set points of the MVs. An OPC-UA communication network is used for this purpose. Fig. 7 shows the NMPC architecture for controlling the grinding circuit.

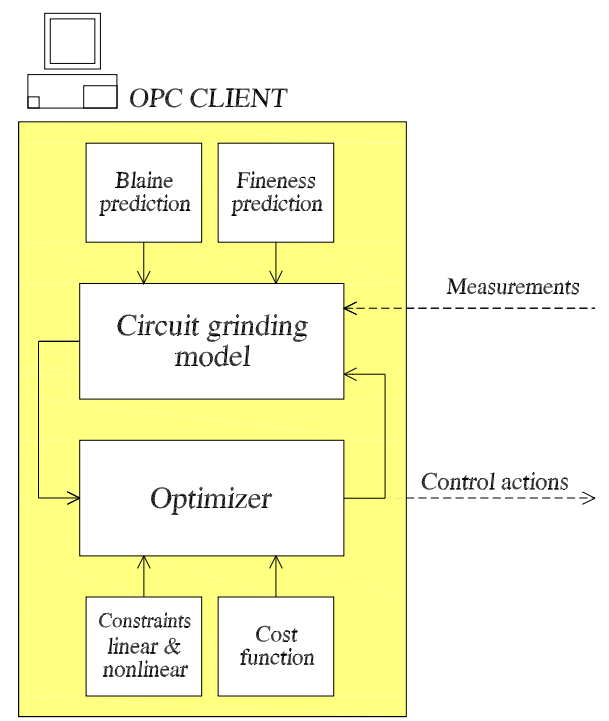

Fig. 7. The NMPC architecture for a centralized grinding circuit controller

Selecting the cost function for the NMPC could be a challenging task. In the development of this application it is required to control the cement fineness through a userconfigurable set point of the Blaine. Therefore, the Blaine needs to be included in the cost function of the optimal control problem (OCP). The Blaine is not measured online and needs a tool for predicting its value, which is developed by using the following equation [13]:

$$
\text { Blaine }=\sum_{i=1}^{n} \frac{6 f_{3 i}}{\rho\left(x_{i+1}-x_{i}\right)} \ln \frac{x_{i+1}}{x_{i}}
$$

where,

$x_{i} \quad-\quad$ Lower size boundary of fraction $i$

$x_{i+1} \quad-\quad$ Upper size boundary of fraction $i$

In consequence, the OCP is defined as follows: $\min$

s.t.

$$
J_{N}\left(\mathbf{f}_{0}, \mathbf{u}(\cdot)\right)=\sum_{k=0}^{N-1}\left|\left[\begin{array}{c}
\text { Reject flow }\left(B_{2}\right) \\
\text { Blaine }
\end{array}\right]-\mathbf{r}\right|
$$

$$
\begin{aligned}
{\left[{ }^{\mathbf{u}} \mathbf{F}\right]_{k+1} } & =\left[\begin{array}{c}
\mathbf{f}_{1}(k+1) \\
\mathbf{f}_{2}(k+1) \\
\mathbf{f}_{3}(k+1)
\end{array}\right]=\mathbf{g}\left(\left[{ }^{\mathbf{u}} \mathbf{F}\right]_{k}, \mathbf{u}_{k}\right) \\
{[\mathbf{F}]_{k} } & \in \mathbb{X}_{x_{\min }}^{x_{\max }} \subset \mathbb{X} \\
\mathbf{u}_{k} & \in \mathbb{U}_{u_{\min }^{u_{\max }} \subset \mathbb{U}}
\end{aligned}
$$

where $|\cdot|$ represents the norm of a matrix, $\left[{ }^{\mathbf{u}} \mathbf{F}\right]$ is the prediction matrix of the state vectors when the calculated control signal $\mathbf{U}=\{\mathbf{u}(1), \mathbf{u}(1), \ldots, \mathbf{u}(N)$,$\} is applied;$ $\mathbf{r}=\left[\begin{array}{ll}B 2_{\text {ref }} & \text { Blaine }_{\text {ref }}\end{array}\right]^{T}$ is the reference vector containing the desired reject flow in $t p h$, and the desired cement fineness $\left(\mathrm{cm}^{2} / \mathrm{g}\right)$; and $N$ is the prediction horizon.

The MVs constraints are set according to the grinding circuit operating rules, adapted from the panelists working at the cement plant, which are:

$$
\begin{aligned}
170 \leq u_{1} \leq 200 \\
1000 \leq u_{2} \leq 1100 \\
80 \leq u_{3} \leq \infty
\end{aligned}
$$

The MVs $u_{1}$ and $u_{2}$ of the control vector $\mathbf{u}$ are restricted to be integer, while $u_{3}$ is continuous. Problems of this type are named mixed-integer nonlinear programming (MINLP). The mixed-integer linear, and nonlinear programming package of TOMLAB for MATLAB was used for solving the OCP. TOMLAB implements a branch-and-bound algorithm searching a tree whose nodes correspond to nonlinearly constrained continuous optimization problems. The method avoids the use of penalty functions. Global convergence is enforced through the use of a trust region and a filter that accepts a trial point whenever the objective or the constraint violation is improved compared to all previous iterations.

\section{CONTROLLER IMPLEMENTATION AND OPERATION}

A list of steps for implementing the NMPC algorithm is shown in Algorithm 1. The workstation running the NMPC algorithm operates as an OPC client to communicate bidirectionally with the SCADA system with a sampling time of one minute. Fig. 8 shows the industrial network configuration of the system. The OPC software used for the communication is the Matrikon OPC Tunneller, which allows a transparent communication between the Pregrinding SCADA server, Grinding SCADA server, and the NMPC control server.

Fig. 9 shows the block diagram of the SCADA tags involved in the NMPC algorithm computation. It can be seen (from Fig. 9) that the system implementation allows two operating modes: manual mode, and automatic mode. The manual mode consists of the classic operation of the grinding circuit performed by the operators by applying rules acquired by training and also by experience. The automatic mode initiates the NMPC to take the control of the grinding circuit. 


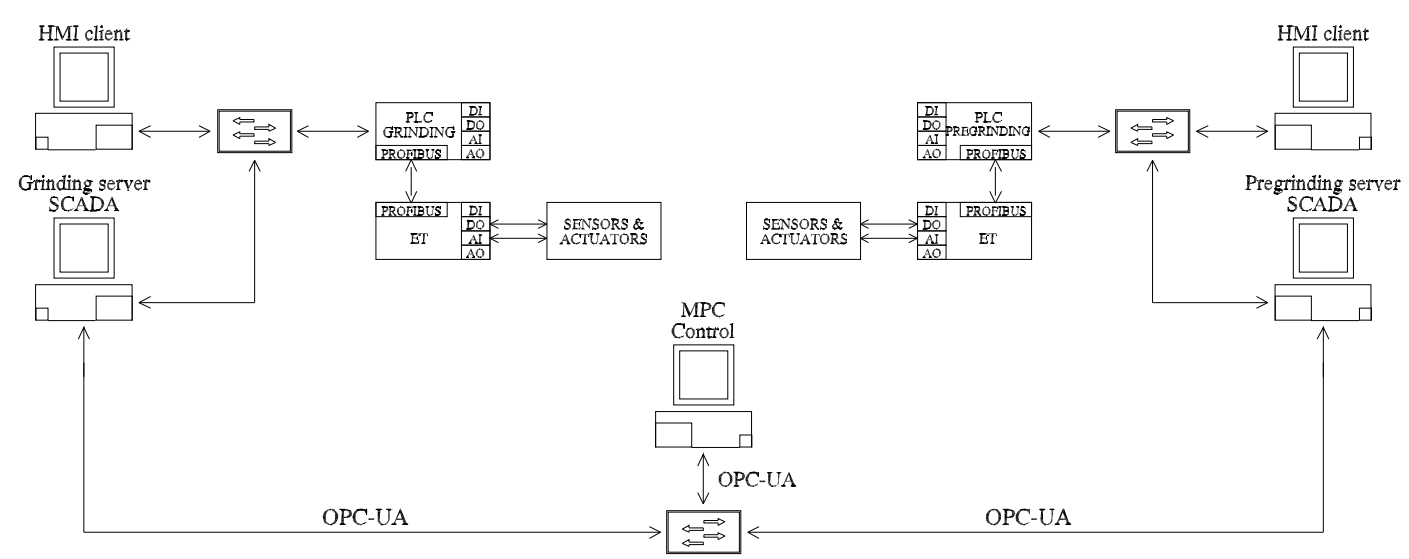

Fig. 8. Network configuration of the system

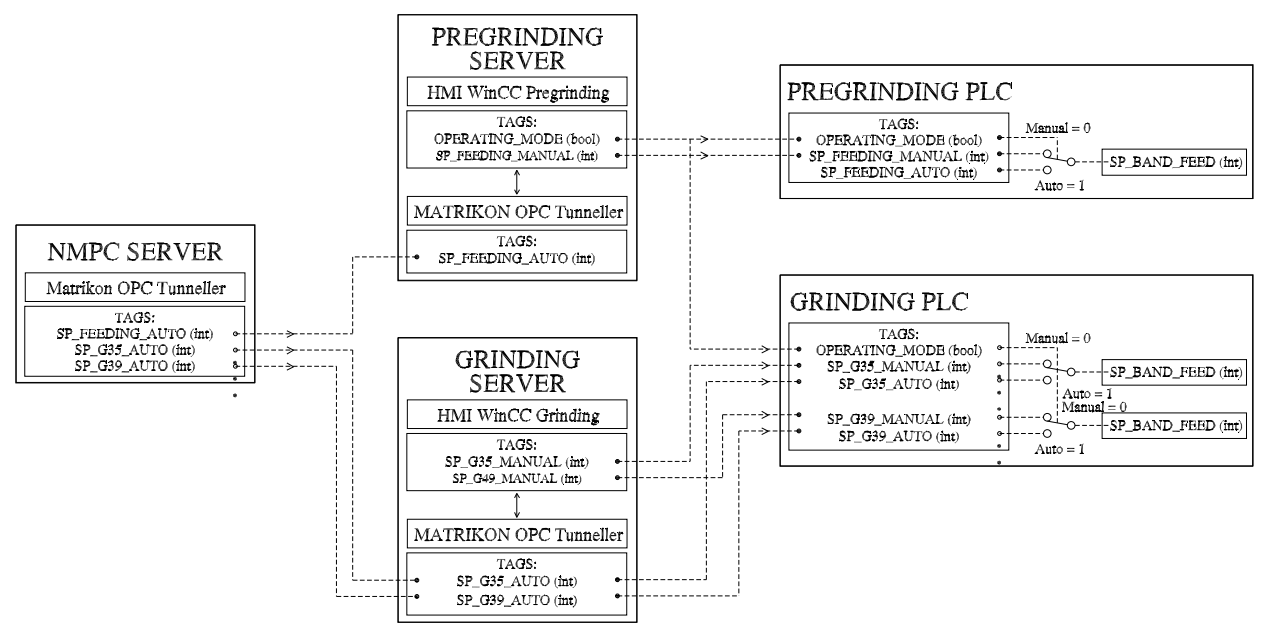

Fig. 9. SCADA tags map of the NMPC implementation

\footnotetext{
Algorithm 1: NMPC algorithm for automatic operation of the grinding circuit

1: Define a vector of references: $\mathbf{r}=\left[\begin{array}{cc}B 2_{\text {ref }} & \text { Blaine }_{\text {ref }}\end{array}\right]^{T}$

2: Take system measurements:

$$
\begin{aligned}
& \mathbb{Z}_{k}=\left[\begin{array}{ccc}
B_{0} & B_{2} & \text { Blaine }
\end{array}\right]^{T} \\
& \quad{ }^{*} \text { Laboratory tests of the fineness are performed every two hours }
\end{aligned}
$$
}

3: Solve the OCP of Eq. (18)

4: Define the control law $\mu\left(\mathbb{Z}_{k}\right)=\mathbf{u}^{*}(1)$

Fig. 10 shows results of the grinding circuit operation in manual and automatic mode. The dashed vertical red line indicates the instant of time when the NMPC is turned on. The reject flow reference $\left(B 2_{\text {ref }}\right)$ is set to $85 \mathrm{tph}$, and after four hours of operation of the grinding circuit a step change to a new reference of $75 \mathrm{tph}$ is applied for a period of four hours. Finally, the reference for $B_{2}$ is set back to $85 \mathrm{tph}$. The reject flow shows a stable behavior with low steady-state error, when the grinding circuit is operated by the NMPC, which is not the case when the system is operated manually.

During the manual operation of the grinding circuit, the energy consumption as well as the cement fineness are not optimal. The energy consumption of the grinding circuit is highly related with the circulating load $(C L)$. The CL defines the number of times a raw particle passes through the grinding circuit before becoming cement, and is calculated as follows:

$$
C L=\frac{B_{0}+B_{2}}{B_{0}}
$$

where $B_{0}$ represents the fresh feed flow in $t p h$, and $B_{2}$ represents the reject flow in $t p h$.

Typically, a good practice in operating the grinding circuit is performed by keeping $2.0 \leq C L \leq 2.5$. Fig. 11 shows the $C L$ and the Blaine of the grinding circuit prior and during the operation of the NMPC. The cement fineness variation is low and is kept close to the reference value of $4100\left(\frac{\mathrm{cm}^{2}}{\mathrm{~g}}\right)$. The $C L$ is also kept close to 2.0 , which means a reduced energy consumption than the manual operation of the grinding circuit.

\section{SYSTEM LIMITATIONS}

The Markovian model was estimated with experimental data obtained from laboratory tests of three streams of the grinding circuit. The Markovian model assumes that there are no substantial variations in the composition of the fresh feed, i.e. added percentages of pozzolana, gypsum, etc., are kept 

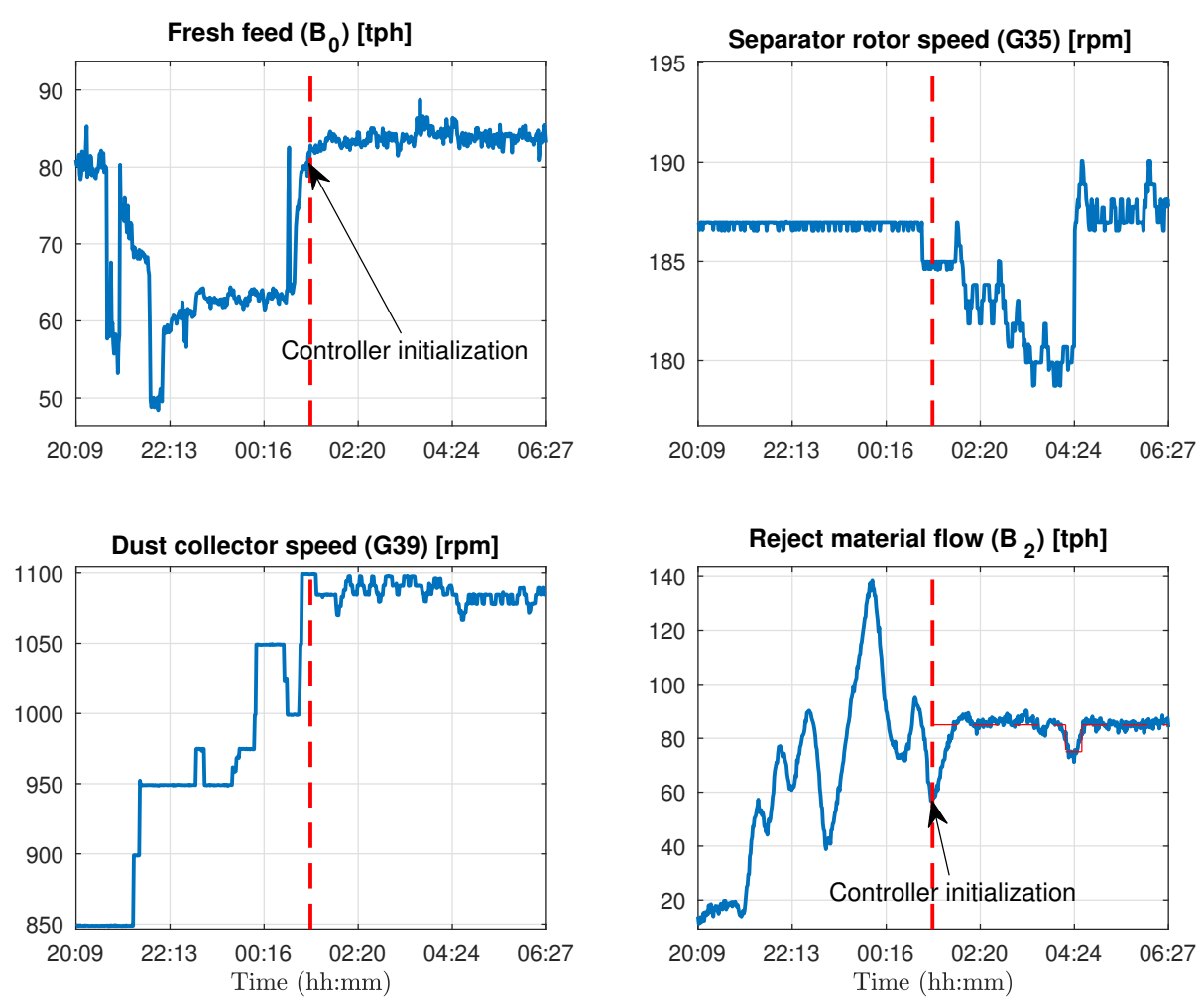

Fig. 10. MVs and $B_{2}$ response prior and after the operation of the NMPC in the grinding circuit
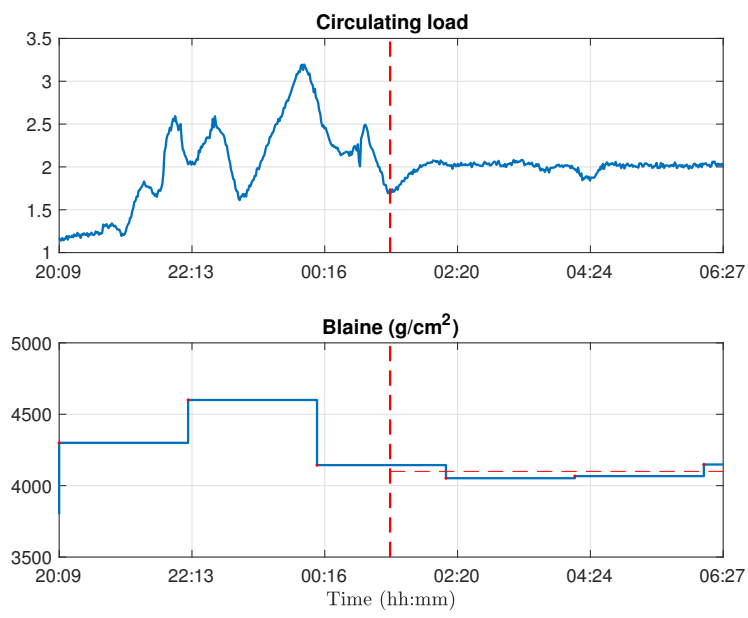

Fig. 11. $C L$ and Blaine responses prior and during the operation of the grinding circuit with the NMPC algorithm

fixed. In the event of a substantial change in the product recipe; for instance, gypsum lack or pozzolana percentage variation greater than $10 \%$, the grinding circuit model fails to correctly predict the system behavior.

Fig. 12 shows the events of failure just described. Around $12 \mathrm{~h} 00$ there is a gypsum lack, and thirty minutes later there is a substantial variation of the percentage of pozzolana in the feeding. The proposed control system is unable to keep a stable operation of grinding circuit while the non-modeled events are present. To avoid harmful calculations of the NMPC
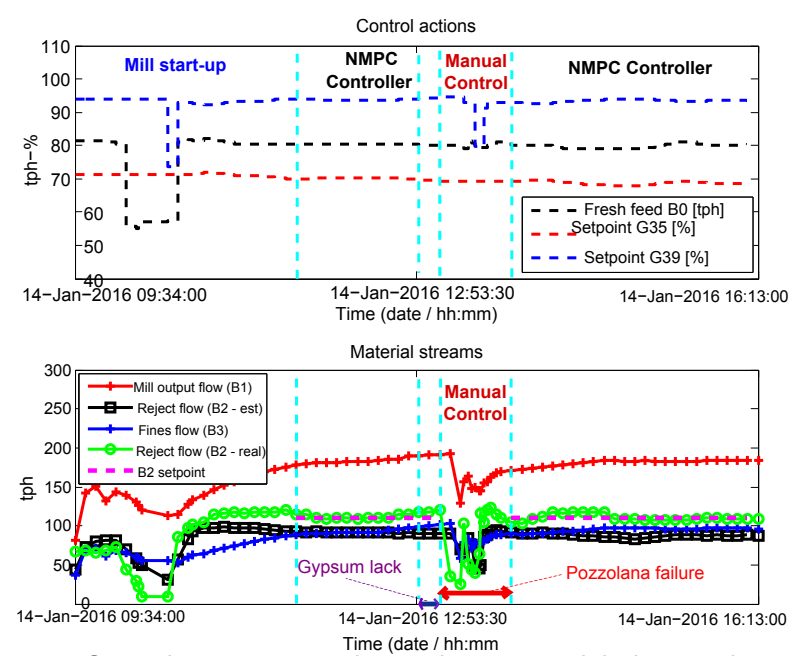

Fig. 12. Control system operation under non-modeled scenarios

algorithm, although the OCP constraints protect the systems integrity, when the estimated value of the reject flow in $t p h$ differs from the measured $B_{2}$ for five consecutive samples causing a mean squared error greater than 1 , manual control is set. Afterwards, the operator has to acknowledge an alarm and correct the system failure before turning on the NMPC.

The industry also has developed solutions for similar problems as the one solved in this paper. Reference [19] shows a detailed survey of industrial MPC technology, from which we have extracted some features of the most known MPC technologies in the market, to establish a comparison with the proposed solution by the authors, as shown in Table II. 
TABLE II

SYSTEM COMPARISON WITH COMMERCIAL PRODUCTS

\begin{tabular}{|c|c|c|c|}
\hline Name & Manufacturer & Modelization & $\begin{array}{l}\text { Optmization } \\
\text { method }\end{array}$ \\
\hline Aspentech & Aspentech & $\begin{array}{l}\text { Multivariate } \\
\text { step response }\end{array}$ & $\begin{array}{l}\text { Linear } \\
\text { programming }\end{array}$ \\
\hline RMCPT & Honeywell & $\begin{array}{l}\text { Multivariable } \\
\text { ARX model }\end{array}$ & $\begin{array}{l}\text { Quadratic } \\
\text { programming }\end{array}$ \\
\hline Connoisseur & Sim Sciences & $\begin{array}{l}\text { Recursive } \\
\text { ARX model }\end{array}$ & $\begin{array}{l}\text { Quadratic } \\
\text { programminc }\end{array}$ \\
\hline 3dMPC & $\mathrm{ABB}$ & State-space & Not available \\
\hline NMPC & Authors & Markov chain & MINLP \\
\hline
\end{tabular}

\section{CONCLUSIONS}

This paper presents the design and implementation of a supervisory control strategy, based on the NMPC algorithm, to operate a grinding circuit in cement industry. Significant performance improvement is achieved with the use of the proposed control strategy over the manual operation of the grinding circuit. The control system keeps the material flows stabilized, as well as the cement fineness, and the $C L$ within a recommended operating range to reduce energy consumption. The energy savings reached in the cement plant were directly reflected in the montly energy bills, with reductions up to $\$ 7 \mathrm{~K}$. Although the reduction suggests a good result, it also reflects that the prior operation of the grinding circuit (manual operation) was far from optimal.

The proposed controller keeps the mill feed $\left(B_{0}\right)$ above the configurable constraint of $80 \mathrm{tph}$, which represented an increase in the productivity of the final product.

The control system was implemented to interact with the already existing SCADA system by using the OPC communication standard. For all the operational tests, there was not any packet loss during the bidirectional communication. The OPC communication network can also be used for other purposes, such as data storage of PV and MV in a remote database.

\section{REFERENCES}

[1] N. Madlool, R. Saidur, N. Rahim, and M. Kamalisarvestani, "An overview of energy savings measures for cement industries," Renewable and Sustainable Energy Reviews, vol. 19, pp. 18-29, 2013.

[2] T. Gao, L. Shen, M. Shen, L. Liu, and F. Chen, "Analysis of material flow and consumption in cement production process," Journal of Cleaner Production, vol. 112, pp. 553-565, 2016.

[3] D. C. Tsamatsoulis, "Optimizing the control system of cement milling: Process modeling and controller tuning based on loop shaping procedures and process simulations," Brazilian Journal of Chemical Engineering, vol. 31, no. 1, pp. 155-170, 2014.

[4] G. Muralidharan, M. Chidambaram, B. Recke, and J. B. Jorgensen, "Soft constrained based MPC for robust control of a cement grinding circuit," in Dynamics and Control of Process Systems, vol. 10, pp. 475-480, 2013.

[5] P. Subbaraj and P. S. G. Anand, "Optimal design of a fuzzy logic controller for control of a cement mill process by a genetic algorithm," Instrumentation Science \& Technology, vol. 39, no. 3, pp. 288-311, 2011.

[6] P. Zhou, T. Chai, and J. Sun, "Intelligence-based supervisory control for optimal operation of a DCS-controlled grinding system," IEEE Transactions on Control Systems Technology, vol. 21, no. 1, pp. 162 175, 2013.

[7] J. D. le Roux, R. Padhi, and I. K. Craig, "Optimal control of grinding mill circuit using model predictive static programming: A new nonlinear MPC paradigm," Journal of Process Control, vol. 24, no. 12, pp. 29-40, 2014.
[8] P. Karelovic, E. Putz, and A. Cipriano, "A framework for hybrid model predictive control in mineral processing," Control Engineering Practice, vol. 40, pp. 1-12, 2015.

[9] W. Dai, T. Chai, and S. X. Yang, "Data-driven optimization control for safety operation of hematite grinding process," IEEE Transactions on Industrial Electronics, vol. 62, pp. 2930-2941, May 2015.

[10] R. Lepore, A. V. Wouwer, M. Remy, and P. Bogaerts, "Receding-horizon estimation and control of ball mill circuits," in Assessment and Future Directions of Nonlinear Model Predictive Control, pp. 485-493, Berlin, Heidelberg: Springer Berlin Heidelberg, 2007.

[11] M. Boulvin, A. Wouwer, R. Lepore, C. Renotte, and M. Remy, "Modeling and control of cement grinding processes," IEEE Transactions on Control Systems Technology, vol. 11, no. 5, pp. 715-725, 2003.

[12] O. Häggström, "Finite Markov chains and algorithmic applications," in London Mathematical Society Student Texts, Cambridge University Press, 2001.

[13] G. G. Mejeoumov, "Improved cement quality and grinding efficiency by means of closed mill circuit modeling," 2007. PhD thesis, Texas A \& M.

[14] L. Austin, P. Bagga, and M. Celik, "Breakage properties of some materials in a laboratory ball mill," Powder Technology, vol. 28, no. 2, pp. 235-243, 1981.

[15] H. Berthiaux, "Analysis of grinding processes by Markov chains," Chemical Engineering Science, vol. 55, no. 19, pp. 4117-4127, 2000.

[16] H. Berthiaux, V. Mizonov, and V. Zhukov, "Application of the theory of Markov chains to model different processes in particle technology," Powder Technology, vol. 157, no. 1-3, pp. 128-137, 2005.

[17] L. Grüne and J. Pannek, Nonlinear Model Predictive Control: Theory and Algorithms. Springer, 2011.

[18] J. Maciejowski, Predictive Control with Constraints. Prentice-Hall, 2002.

[19] S. Qin and T. A. Badgwell, "A survey of industrial model predictive control technology," Control Engineering Practice, vol. 11, no. 7, pp. 733-764, 2003 .

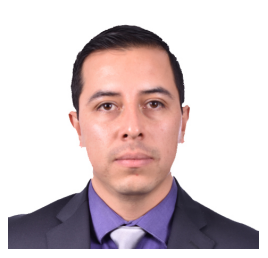

Luis I. Minchala (M'05) received his B.S.E.E. degree in 2006 from the Salesian Polytechnic University in Cuenca, Ecuador, and his M.S., and Ph.D. degrees from Tecnológico de Monterrey, México, in 2011 and 2014, respectively. During summer 2012 to summer 2013 he was a visiting scholar in Concordia University in Montreal, Canada. From 2015 Dr. Minchala is a full time researcher with the department of electrical, electronic and telecommunications engineering at Universidad de Cuenca. Currently Dr. Minchala is a posdoctoral fellow at Tecnológico de Monterrey.

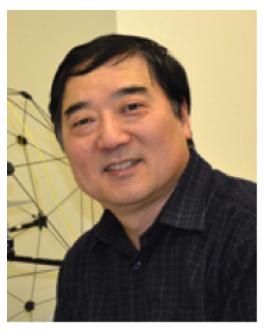

Youmin Zhang (SM'07) received the B.S., M.S., and Ph.D. degrees with a specialization in automatic controls from Northwestern Polytechnical University, Xian, China, in 1983, 1986, and 1995, respectively. Dr. Zhang is currently a Professor with the Department of Mechanical and Industrial Engineering and the Concordia Institute of Aerospace Design and Innovation, Concordia University, Montreal, Quebec, Canada. He has authored four books, over 380 journal and conference papers, and book chapters.

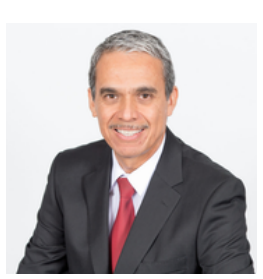

Luis Garza-Castañón (M'08) received his B.S., M.S. and PhD degrees from Tecnológico de Monterrey in Monterrey, NL, México in 1986, 1988 and 2001, respectively. Dr. Garza is currently an Associate Professor with the Mechatronics and Automation Department at Tecnológico de Monterrey, Monterrey, NL, México. He has authored more than 90 journal and conference papers, and book chapters. 